\title{
Non-invasive right ventriculo-arterial coupling as a rehospitalization predictor in dilated cardiomyopathy: A comparison of five different methods
}

\author{
Aura Vijîiac ${ }^{1,2}$, Vlad Bătăilă2 , Sebastian Onciul1,2, Violeta Verinceanu' ${ }^{2}$, Claudia Guzu², Silvia Deaconu ${ }^{1,2}$, \\ Ioana Petre ${ }^{1,2}$, Alina Scărlătescu², Diana Zamfir ${ }^{2}$, Maria Dorobanțu' ${ }^{1,2}$ \\ 1"Carol Davila" University of Medicine and Pharmacy, Bucharest, Romania \\ 2Emergency Clinical Hospital, Bucharest, Romania
}

\author{
Correspondence to: \\ Aura Vijîiiac, MD, PhD, \\ Emergency Clinical Hospital, \\ 8, Calea Floreasca, 014491 \\ Bucharest, Romania, \\ phone: +40 215992 300/418 \\ e-mail: \\ aura.apostolescu@yahoo.com \\ Copyright by the Author(s), 2022 \\ DOI: $10.33963 /$ KP.a2021.0190 \\ Received: \\ October 6, 2021 \\ Accepted: \\ December 23, 2021 \\ Early publication date: \\ December 23, 2021
}

\begin{abstract}
A B S TRA C T
Background: Right ventricular (RV) pulmonary artery coupling (RVAC) is a predictor of outcome in left-sided heart failure (HF). Several echocardiographic estimates for RVPAC have been proposed.

Aims: This study aimed to compare multiple non-invasive methods to calculate RVPAC and to assess its prognostic role in patients with dilated cardiomyopathy (DCM).

Methods: We prospectively enrolled 60 stable patients with DCM. RVPAC was estimated using five methods: as the tricuspid annular plane excursion/pulmonary artery systolic pressure (PASP) ratio; as the RV global longitudinal strain/PASP ratio; as the RV free wall strain (RVFW-LS)/PASP ratio; as the three-dimensional (3D) RV ejection fraction (RVEF)/PASP ratio; and as the 3D RV stroke volume (SV)/end-systolic volume (ESV) ratio. Patients were followed for a mean period of 18 (9) months for the endpoint of HF rehospitalizations.
\end{abstract}

Results: Twenty-nine patients (48\%) reached the endpoint. All RVPAC estimates were more impaired in those patients reaching the endpoint $(P<0.001$ for all) and all predicted rehospitalizations in unadjusted analysis. RVFW-LS/PASP and RVEF/PASP remained independent predictors of events, after adjustment for clinical and echocardiographic confounders. Using cut-offs obtained from receiver operating characteristic (ROC) analysis, we found that patients with RVFW-LS/PASP $>-0.40$ and patients with RVEF/PASP $<1.30$ had a higher risk of HF rehospitalization (log-rank $P=0.001$ and $P=0.002$, respectively).

Conclusion: RVFW-LS/PASP and RVEF/PASP as non-invasive estimates of RVPAC are independent predictors of HF rehospitalization in patients with DCM.

Key words: dilated cardiomyopathy, right ventricular-pulmonary artery coupling, RVEF/PASP ratio, RVFW-LS/PASP ratio

\section{INTRODUCTION}

The prognostic role of right ventricular (RV) dysfunction in various cardiovascular diseases is well established [1-3]. While impaired RV performance is a recognized outcome predictor in left-sided heart disease [4, 5], there is growing interest regarding the role of right ventriculo-vascular interplay in patients with heart failure (HF) $[6,7]$. RV-pulmonary artery coupling (RVPAC) characterizes the interaction between ventricular contractility and its afterload, thus allowing the evaluation of the RV and pulmonary circulation as an anatomically and functionally interconnected system [8]. The gold standard for RVPAC measurement is the ratio between end-systolic RV elastance and pulmonary arterial elastance, derived from invasive pressure-volume loops [9]. However, RVPAC can be estimated non-invasively using echocardiography and several surrogate parameters [10-13].

We hypothesized that right ventriculo-vascular decoupling is related to the risk of rehospitalization for HF in patients with dilated 
WHAT'S NEW?

In our study, we compared five different methods for the non-invasive estimation of right ventricular-pulmonary artery coupling (RVPAC) in patients with dilated cardiomyopathy. Patients with exacerbation of heart failure requiring hospitalization had significantly more impaired RVPAC. Among the different surrogates of RVPAC, the only two independent event predictors were the ratio of right ventricular free wall strain to pulmonary artery systolic pressure, and the ratio of three-dimensional right ventricular ejection fraction to pulmonary artery systolic pressure.

cardiomyopathy (DCM). We sought to assess the prognostic role of RVPAC in this setting while comparing different non-invasive methods for RVPAC estimation — using both conventional and advanced echocardiographic techniques such as three-dimensional (3D) and speckle-tracking echocardiography (STE).

\section{METHODS}

\section{Study population}

We prospectively enrolled consecutive outpatients with non-ischemic DCM who were referred to our echocardiography department between January 2019 and December 2019. DCM was defined [14] based on the following criteria: (1) dilated left ventricle (LV), according to cut-offs from the current guidelines of chamber quantification [15]; (2) Simpson biplane LV ejection fraction (LVEF) $<40 \%$; and (3) absence of significant coronary artery disease (defined as $>70 \%$ stenosis of a major epicardial vessel). Since the right heart is highly dependent on loading conditions, we only included patients that were clinically and hemodynamically stable, with no change in diuretic dose in the two weeks before enrollment. We excluded patients with poor acoustic window, atrial fibrillation, or inability to hold the breath (which would have hampered 3D acquisitions), severe tricuspid regurgitation (TR), and cor pulmonale. Sixty patients formed the final study cohort. Investigators collected demographic and clinical data, including cardiovascular risk factors, a New York Heart Association (NYHA) class, and brain natriuretic peptide (BNP) levels, when available. The study protocol, complying with the Declaration of Helsinki, was approved by the local ethics committee of the Emergency Clinical Hospital of Bucharest, and all patients provided written informed consent at enrollment.

For our study, the endpoint was the first episode of HF exacerbation requiring hospitalization. Patients were prospectively followed by regular clinical visits and phone contact to ascertain the occurrence of the main endpoint, and the time of the first rehospitalization was used in our survival analysis. The follow-up was conducted for 18 (9) months.

\section{Echocardiographic assessment}

We performed comprehensive two-dimensional (2D) echocardiographic examinations for all patients with a Vivid GE
Vingmed E9 ultrasound machine equipped with an M5S probe. Offline data analysis was done using dedicated software (EchoPAC BT 12, General Electric Healthcare, Milwaukee, WI, USA). For the LV, we assessed the dimensions, systolic and diastolic function according to current recommendations [16]. For the RV, we measured conventional parameters of systolic function such as tricuspid annular plane systolic excursion (TAPSE), peak systolic tissue Doppler velocity of the tricuspid annulus (S wave), and RV fractional area change (RV-FAC) from apical RV-focused view, as recommended $[16,17]$.

For RV strain, we used high frame-rate acquisitions and we manually traced the RV endocardial border at end-systole. Readjustments were made if needed, including in the region of interest, the whole $\mathrm{RV}$ wall, but excluding the pericardium. EchoPAC - Q Analysis software automatically divided the RV into six segments, three for the RV free wall (RVFW), and three for the interventricular septum (IVS). We then calculated the global longitudinal strain of the RV (GLS-RV) as the mean of the six segments, and the longitudinal strain of the RVFW (RVFW-LS) as the mean of the three RVFW segments, as recommended $[18,19]$. Normal strain values are negative [18] because it is a measure of myocardial shortening; thus, values that are less negative reflect impaired myocardial shortening.

For estimation of pulmonary artery systolic pressure (PASP), we used the maximal velocity of the TR jet $\left(\mathrm{TRV}_{\max }\right)$ obtained from the continuous wave Doppler spectrum and right atrial pressure (RAP): PASP $=4 \times \mathrm{TRV}_{\max }{ }^{2}+\mathrm{RAP}$. RAP was estimated based on the inferior vena cava diameter and respiratory collapsibility, as described in guidelines [16].

Using a $4 \mathrm{~V}$ probe, we performed six-beat full-volume 3D acquisitions, with electrocardiographic gating during apnea. We used the apical RV-focused view for 3D data sets acquired for the assessment of the RV [20]. We performed offline image post-processing and reconstruction using 4D RV-Function software (TomTec Imaging Systems, Unterschleissheim, Germany). After tracing the endocardial surface of the RV at both end-systole and end-diastole, the software generated the RV stroke volume (SV), ejection fraction (RVEF), end-diastolic and end-systolic volume (ESV) (Figure 1). Interand intra-observer reproducibility for RV strain and RVEF in our laboratory has been recently published [5]. 


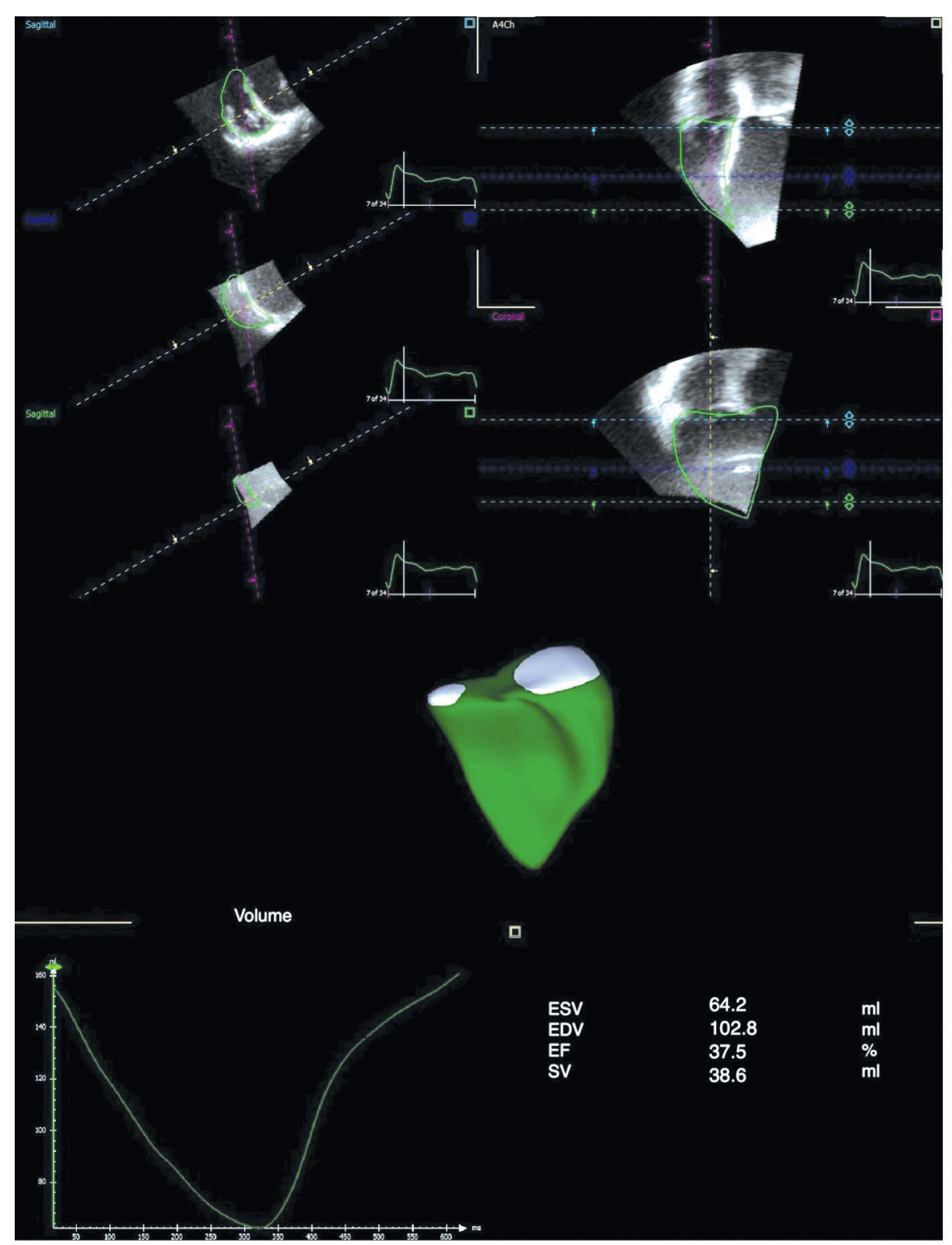

Figure 1. Three-dimensional volumetric assessment of the right ventricle using dedicated software

Abbreviations: ESV, end-systolic volume; $E D V$, end-diastolic volume; $E F$, ejection fraction; SV, stroke volume

\section{Non-invasive estimation of RVPAC}

We estimated the RVPAC using five different methods, all of which were previously proposed and studied:

- as the TAPSE/PASP ratio [10, 21];

- as the GLS-RV/PASP ratio [12, 22];

- as the RVFW-LS/PASP ratio [12, 23];

- as the 3D RVEF/PASP ratio [11]; and

- as the 3D SV/ESV ratio $[24,25]$.

\section{Statistical analysis}

All analysis was performed with SPSS version 20.0 statistical software package. The Kolmogorov-Smirnov test was used to check whether variables were normally distributed. Continuous data were expressed as mean (standard deviation [SD]) if normally distributed, or as median and interquartile range otherwise, and compared with Student's t-test or the Mann-Whitney $\mathrm{U}$ test (as dictated by distribution). Pearson's correlation coefficient was used to assess cor- relations between continuous variables. Categorical data were expressed as numbers and percentages, and they were compared using the test.

We used receiver operating characteristic (ROC) curves and the respective area under the curve (AUC) to identify optimal cut-off values for event prediction, based on the highest sum of sensitivity and specificity. Using these cut-offs, we performed Kaplan-Meier analysis for event-free survival, and we compared survival curves with the log-rank test. We performed Cox proportional hazards regression to determine the prognostic power of non-invasive RVPAC. Results were reported as hazard ratios (HR) with $95 \%$ confidence intervals $(\mathrm{Cl})$. We constructed the multivariable model choosing covariates that have clinical relevance and are well-established event predictors in DCM: age, an NYHA class, LVEF, and the mitral E/E' ratio. Statistical significance was defined as a two-tailed $P$-value $<0.05$. 
Table 1. Basic characteristics

\begin{tabular}{|c|c|c|c|c|}
\hline Variables & $\begin{array}{l}\text { Overall } \\
(n=60)\end{array}$ & $\begin{array}{l}\text { Hospitalized } \\
\qquad(n=29)\end{array}$ & $\begin{array}{l}\text { Not hospitalized } \\
\qquad(n=31)\end{array}$ & $P$-value \\
\hline Age, years & $61(14)$ & $61(14)$ & $60(14)$ & 0.79 \\
\hline Men, n (\%) & $40(67)$ & $19(66)$ & $21(68)$ & 0.86 \\
\hline Systolic BP, mm Hg & $124(13)$ & $122(13)$ & $125(12)$ & 0.32 \\
\hline Diastolic BP, mm Hg & $75(11)$ & $72(10)$ & $77(11)$ & 0.04 \\
\hline Heart rate, bpm & $78(15)$ & $80(17)$ & $77(13)$ & 0.44 \\
\hline NYHA class, n (\%) & & & & 0.003 \\
\hline 1 & $2(3)$ & $0(0)$ & $2(6)$ & \\
\hline II & $27(45)$ & $7(24)$ & $20(65)$ & \\
\hline III & $25(42)$ & $17(59)$ & $8(26)$ & \\
\hline IV & $6(10)$ & $5(17)$ & $1(3)$ & \\
\hline \multicolumn{5}{|l|}{ Comorbidities, n (\%) } \\
\hline Hypertension & $41(68)$ & $17(59)$ & $24(77)$ & 0.12 \\
\hline Diabetes mellitus & $11(18)$ & $6(21)$ & $5(16)$ & 0.65 \\
\hline Smoking & $19(32)$ & $8(28)$ & $11(35)$ & 0.51 \\
\hline CKD & $37(62)$ & $19(66)$ & $18(58)$ & 0.55 \\
\hline \multicolumn{5}{|l|}{ Medication, n (\%) } \\
\hline ACE-I/ARBs/ARN-I & $57(95)$ & $28(97)$ & $29(94)$ & 0.59 \\
\hline$\beta$-blocker & $59(98)$ & $28(97)$ & $31(100)$ & 0.48 \\
\hline MRA & $58(97)$ & $29(100)$ & $29(94)$ & 0.49 \\
\hline Loop diuretic & $41(68)$ & $24(83)$ & $17(55)$ & 0.02 \\
\hline Digoxin & $11(18)$ & $8(28)$ & $3(10)$ & 0.07 \\
\hline BNP levels, pg/ml & $478(286-910)$ & 703 (404-1080) & $388(204-535)$ & 0.005 \\
\hline
\end{tabular}

Continuous data are expressed as mean (standard deviation [SD]) or median (interquartile range [IQR]), depending on the distribution. Categorical data are expressed as number (percentage)

Abbreviations: ACE-I, angiotensin-converting enzyme inhibitor; ARB, angiotensin receptor blocker; ARN-I, angiotensin receptor neprilysin inhibitor; BP, blood pressure; BNP, brain natriuretic peptide; $n$, number of patients; NYHA, New York Heart Association; CKD, chronic kidney disease; MRA, mineralocorticoid receptor antagonist

\section{RESULTS}

\section{Study population}

The mean age in the study group was 61 (14) years and the majority (67\%) were men. During the mean follow-up of 18 (9) months, 29 patients (48\%) reached the main endpoint, being at least once hospitalized for an exacerbation of HF. Twelve patients (20\%) were admitted more than once for decompensated HF during the follow-up. At the end of the follow-up period, there was a total number of 52 rehospitalizations for HF, 4 deaths (all occurring in patients who had already reached the main endpoint), 5 non-fatal cardiac arrests, no heart transplantation, and no ventricular assist device implantation. There was no difference in terms of age and comorbidities between patients reaching the primary endpoint and those who did not (Table 1). Serum BNP was available in 52 (87\%) patients. The prevalence of loop diuretic use, as well as BNP levels, were higher among patients who required rehospitalization $(P=0.02$ and $P=0.005$, respectively).

\section{Echocardiographic data}

All echocardiographic data are summarized in Table 2. While LVEF was similar between patients with and without adverse outcomes $(P=0.17)$, there were significant differences between the two groups in indices reflecting LV diastolic dysfunction. RV function, assessed by both conventional parameters and innovative parameters, was significantly more impaired in patients with adverse outcomes, while PASP was higher in patients with events, with marginal significance $(P=0.049)$. All five estimates of RVPAC were more impaired in patients with events $(P<0.001$ for all).

\section{The prognostic role of non-invasive RVPAC}

Table 3 shows the univariable and multivariable Cox regression for the primary endpoint of HF rehospitalizations. All RVPAC surrogates were endpoint predictors in unadjusted analysis. LVEF did not emerge as a predictor of hospitalizations in unadjusted analysis $(P=0.12)$. We also performed ROC analysis to assess the ability of RVPAC to predict rehospitalizations. As shown in Figure 2, all RVPAC surrogates outperformed LVEF, and they all had good AUCs (Table 4). However, after adjusting for the clinical and echocardiographic confounders in the multivariable model, the RVFW-LS/PASP ratio and the RVEF/PASP ratio were the only RVPAC estimates that remained independent predictors of rehospitalizations (Table 3). The best cut-off value for predicting outcome was -0.40 for RVFW-LS/PASP (sensitivity $69 \%$, specificity $77 \%$ ) and 1.30 for RVEF/PASP (sensitivity $83 \%$, specificity $65 \%$ ). Kaplan-Meier survival curves stratified by these cut-offs are shown in Figure 3. The risk of rehospitalization was higher in patients with RVFW-LS/PASP over $-0.40(\mathrm{HR}, 3.653 ; 95 \% \mathrm{Cl}, 1.657-8.051 ; P=0.001)$ and in patients with RVEF/PASP less than 1.30 (HR, 3.600; $95 \%$ $\mathrm{Cl}, 1.464-8.854 ; P=0.002)$ in univariable analysis. 
Table 2. Echocardiographic data

\begin{tabular}{|c|c|c|c|c|}
\hline Variables & $\begin{array}{l}\text { Overall } \\
(n=60)\end{array}$ & $\begin{array}{l}\text { Hospitalized } \\
\quad(n=29)\end{array}$ & $\begin{array}{l}\text { Not hospitalized } \\
\qquad(n=31)\end{array}$ & $P$-value \\
\hline \multicolumn{5}{|l|}{ LV parameters } \\
\hline LV end-diastolic diameter, $\mathrm{mm}$ & $66(9)$ & $66(8)$ & $65(10)$ & 0.48 \\
\hline LVEDV, $\mathrm{ml}$ & $225(87)$ & $233(88)$ & $217(88)$ & 0.48 \\
\hline LVESV, ml & $170(76)$ & $179(7)$ & $161(77)$ & 0.38 \\
\hline LVEF, \% & $26(7)$ & $24(7)$ & $27(7)$ & 0.17 \\
\hline Mitral E/E' ratio & $14.3(6.6)$ & $16.6(8.1)$ & $12.1(4.8)$ & 0.008 \\
\hline $\mathrm{LA}$ volume index, $\mathrm{ml} / \mathrm{m}^{2}$ & $45(24)$ & $56(27)$ & $34(15)$ & $<0.001$ \\
\hline \multicolumn{5}{|l|}{ RV parameters } \\
\hline $\mathrm{RV}$ basal diameter, $\mathrm{mm}$ & $37(33-42)$ & $38(35-47)$ & $34(31-37)$ & 0.002 \\
\hline Tricuspid E/E' ratio & $5.8(2.9)$ & $6.4(3.3)$ & $5.3(2.4)$ & 0.13 \\
\hline TAPSE, mm & $18(4)$ & $16(4)$ & $20(4)$ & $<0.001$ \\
\hline $\mathrm{S}$ wave velocity, $\mathrm{cm} / \mathrm{s}$ & $11.1(2.8)$ & $9.8(2.7)$ & $12.4(2.2)$ & $<0.001$ \\
\hline RV-FAC, $\%$ & $33(12)$ & $31(12)$ & $35(11)$ & 0.12 \\
\hline GLS-RV, \% & $-12.2(5.3)$ & $-9.8(4.6)$ & $-14.4(5.0)$ & $<0.001$ \\
\hline RVFW-LS, \% & $-14.8(9.3)$ & $-11.1(7.2)$ & $-18.2(10.1)$ & 0.003 \\
\hline 3D RVEDV, $\mathrm{ml}$ & $139(53)$ & $140(56)$ & $139(52)$ & 0.94 \\
\hline 3D RVESV, $\mathrm{ml}$ & $80(31)$ & $88(35)$ & $72(25)$ & 0.051 \\
\hline 3D RV SV, ml & $60(31)$ & $52(25)$ & $67(34)$ & 0.06 \\
\hline 3D RVEF, \% & $42(10)$ & $37(9)$ & $47(9)$ & $<0.001$ \\
\hline PASP $(\mathrm{mm} \mathrm{Hg})$ & $39(17)$ & $44(16)$ & $35(17)$ & 0.049 \\
\hline \multicolumn{5}{|l|}{ RVPAC estimates } \\
\hline TAPSE/PASP & $0.56(0.28)$ & $0.43(0.21)$ & $0.68(0.29)$ & $<0.001$ \\
\hline GLS-RV/PASP & $-0.37(0.22)$ & $-0.26(0.17)$ & $-0.47(0.21)$ & $<0.001$ \\
\hline RVFW-LS/PASP & $-0.45(0.38)$ & $-0.27(0.36)$ & $-0.61(0.32)$ & $<0.001$ \\
\hline RVEF/PASP & $1.28(0.61)$ & $0.97(0.38)$ & $1.57(0.65)$ & $<0.001$ \\
\hline RV SV/ESV & $0.80(0.36)$ & $0.62(0.24)$ & $0.96(0.38)$ & $<0.001$ \\
\hline
\end{tabular}

Data are expressed as mean (standard deviation [SD]) or median (interquartile range [IQR]), depending on the distribution Abbreviations: see Figures 1 and 2

Table 3. Univariable and multivariable Cox regression analysis

\begin{tabular}{l|c|c|c|}
\multirow{2}{*}{$\begin{array}{c}\text { Variables } \\
\text { (per unit increase) }\end{array}$} & \multicolumn{2}{c}{ Unadjusted $^{\text {Adjusted }^{\mathrm{a}}}$} \\
\cline { 2 - 4 } & HR (95\% CI) & P-value & HR (95\% Cl) \\
\hline TAPSE/PASP & $0.050(0.008-0.302)$ & 0.001 & $0.158(0.019-1.280)$ \\
GLS-RV/PASP & $31.193(4.337-224.363)$ & 0.001 & $8.215(0.966-69.889)$ \\
RVFW-LS/PASP & $5.010(2.090-11.964)$ & $<0.001$ & $3.122(1.135-8.584)$ \\
RVEF/PASP & $0.245(0.109-0.553)$ & 0.001 & $0.381(0.147-0.988)$ \\
RV SV/ESV & $0.069(0.014-0.347)$ & 0.001 & $0.183(0.032-1.033)$ \\
\hline
\end{tabular}

adjusted for age, NYHA class, LVEF, mitral E/E' ratio

Abbreviations: see Figures 1 and 2

Table 4. AUC for parameters to identify the risk of rehospitalization

\begin{tabular}{|c|c|c|c|}
\hline Parameter & AUC $(95 \% \mathrm{Cl})$ & $P$-value & Cut-off \\
\hline TAPSE/PASP & $0.756(0.635-0.877)$ & 0.001 & 0.47 \\
\hline GLS-RV/PASP & $0.784(0.667-0.901)$ & $<0.001$ & -0.37 \\
\hline RVFW-LS/PASP & $0.766(0.646-0.885)$ & $<0.001$ & -0.40 \\
\hline RVEF/PASP & $0.782(0.667-0.897)$ & $<0.001$ & 1.30 \\
\hline RV SV/ESV & $0.789(0.673-0.904)$ & $<0.001$ & 0.65 \\
\hline LVEF & $0.606(0.461-0.752)$ & 0.158 & 24.2 \\
\hline
\end{tabular}

Abbreviations: see Figures 1 and 2 


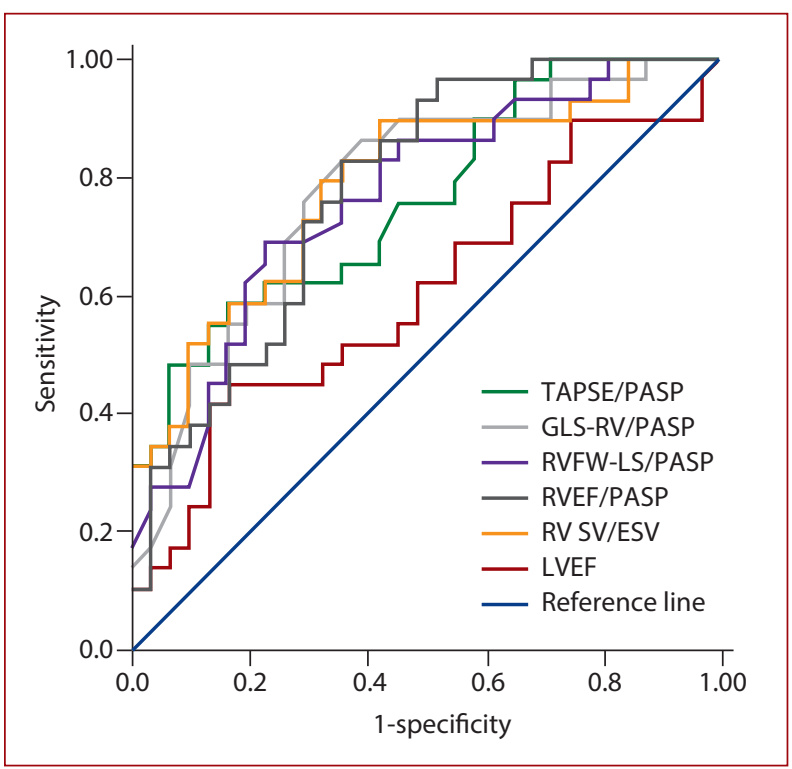

Figure 2. Receiver-operating characteristic analysis of RVPAC and LVEF for the prediction of rehospitalizations

Abbreviations: GLS-RV, global longitudinal strain of the right ventricle; LVEF, left ventricular ejection fraction; PASP, pulmonary artery systolic pressure; ROC, receiver operating characteristic; RV, right ventricle; RVPAC, right ventricular-pulmonary artery coupling; RVFW-LS, longitudinal strain of the right ventricular free wall; SV, stroke volume; TAPSE, tricuspid annular plane systolic excursion; other - see Figure 1

We also tested the constituents of the RVFW-LS/PASP and RVEF/PASP ratios in the same multivariable model. RVEF independently predicted HF hospitalizations (HR, $0.951 ; 95 \% \mathrm{Cl}, 0.906-0.999 ; P=0.046)$, while RVFW-LS and PASP did not (HR, 1.037; 95\% Cl, 0.996-1.080, $P=0.08$ and $\mathrm{HR}, 1.011 ; 95 \% \mathrm{Cl}, 0.988-1.035 ; P=0.35$, respectively).

\section{DISCUSSION}

The findings of our study can be summarized as follows: (1) non-invasive RVPAC was significantly more impaired in patients with DCM who were rehospitalized for HF exacerbation, irrespective of the method used for RVPAC estimation; (2) the RVFW-LS/PASP and RVEF/PASP ratios were the only RVPAC estimates that remained independent predictors of hospitalization; (3) RVFW-LS/PASP > -0.40 and RVEF/PASP $<1.30$ were proposed as cut-offs for predicting a high risk of HF hospitalization.

In DCM, pulmonary hypertension develops as a direct consequence of increased left-sided filling pressures. Initially, the right ventricle will adapt by hypertrophy and remodeling, which will allow for an initial increase in contractility. As the disease progresses, RV maladaptation occurs, and the RV begins to dilate, leading to an impairment of its ejection force and ventriculo-vascular mismatch [26]. This loss of mechanical efficiency of RV contraction in relation to its afterload precedes overt right HF [27]. Hence, early identification of right ventriculo-vascular decoupling would potentially detect those patients at risk of develo-

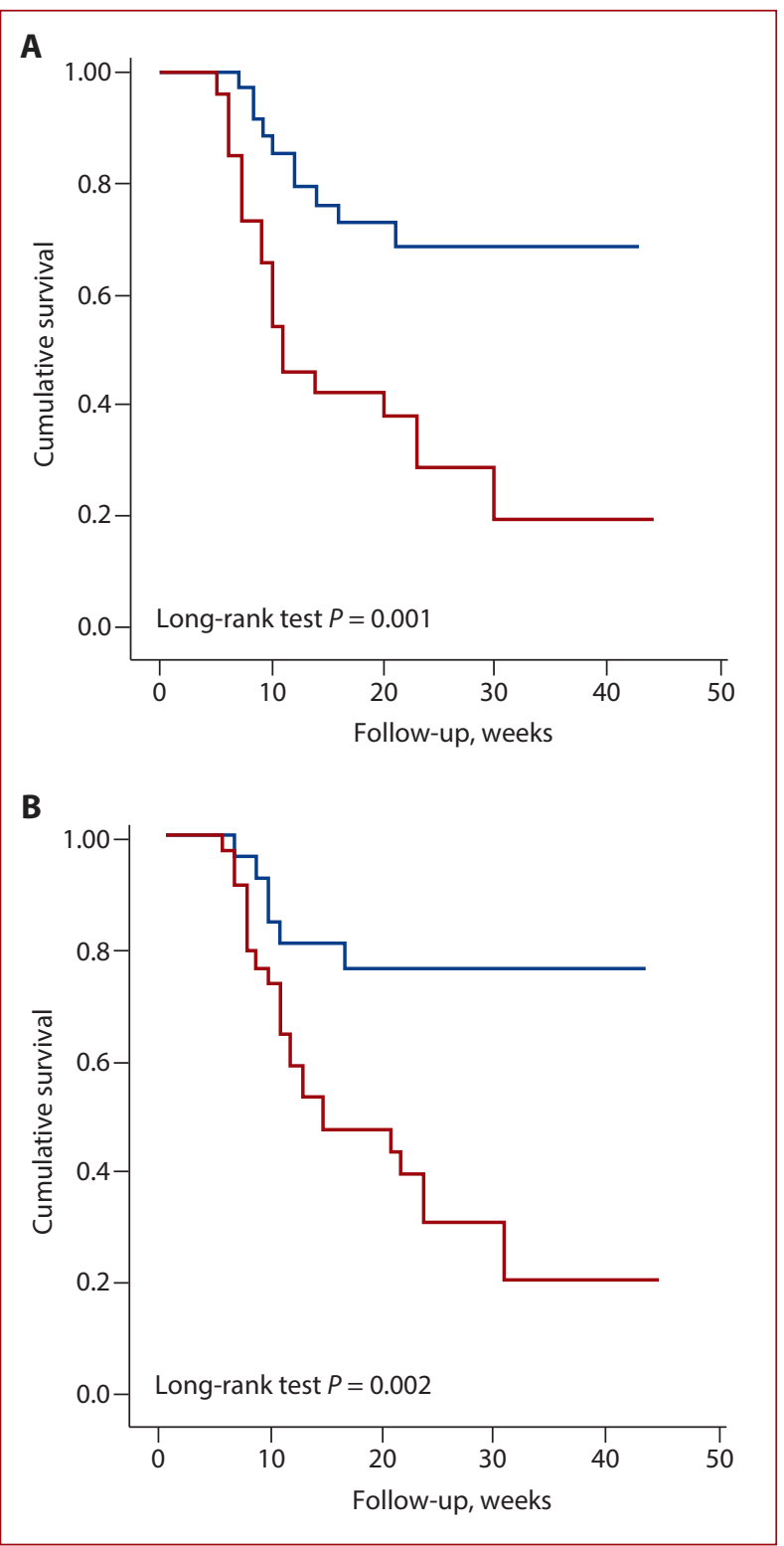

Figure 3. Kaplan-Meier analysis stratified by optimized cut-offs for RVFW-LS/PASP (A) and RVEF/PASP (B)

Abbreviations: RVEF, right ventricular ejection fraction; other - see Figures 1 and 2

ping RV failure [21]. Since RV functional assessment with echocardiography requires a multi-parametric approach [28], several non-invasive estimates for RVPAC have been proposed [10-13, 24]. However, this is the first study to compare the prognostic value of multiple RVPAC estimates in the same population, using parameters derived from both conventional and advanced echocardiography. To our knowledge, this study is also the first to assess RVPAC as an outcome predictor in DCM.

All five RVPAC estimates were outcome predictors in unadjusted analysis in our study. Surprisingly, TAPSE/PASP - the most frequently used surrogate for RVPAC in existing literature $[10,13,21]$ - as well as GLS-RV/PASP lost their predictive power in multivariable analysis. This 
is contrary to the findings of some studies, which found that TAPSE/PASP $[6,10,29]$ and GLS-RV/PASP [12] are independent predictors of events in chronic left-sided HF. The most probable explanation for this discrepancy is that none of these above-mentioned studies included in their multivariable model the mitral E/E' ratio or the left atrial volume. LV diastolic dysfunction is pathophysiologically linked to RV functional impairment [30], so indices of diastolic dysfunction may attenuate the predictive power of RV function in multivariable models. The SV/ESV ratio also lost its predictive role when adjusted for confounders, and it showed weaker correlations with all the other RVPAC surrogates (Table 3). While it was found to have a good correlation with invasive RVPAC in a recent study [24], the prognostic value of the SV/ESV ratio was only tested in a pediatric population with pulmonary hypertension [25], without available data on its role in left-sided HF.

RVFW-LS/PASP remained an independent predictor of HF hospitalizations in our cohort. Similar results were reported by lacoviello et al. [12], who found that RVFW-LS/PASP was an independent predictor of death in chronic HF, in a clinical and echocardiographic multivariable model. However, our study is the first to report this RVPAC surrogate as an independent predictor of outcome after controlling simultaneously for both clinical and echocardiographic risk factors. Moreover, RVFWLS/PASP outperformed RVFW-LS and PASP, which did not independently predict hospitalizations in our study. This suggests that indexing RV function to its afterload provides a better assessment of the interconnected system of the RV and pulmonary artery, and better identification of patients at risk, probably by identifying those patients who are in the phase of transition to overt RV failure [12]. In the current study, we also found RVEF/PASP to be an independent predictor of hospitalizations for DCM. This RVPAC estimate was previously tested by Nochioka et al. [11] in a community-based elderly cohort including both patients with clinically overt HF and at risk of developing $\mathrm{HF}$, and it showed an independent predictive value for HF hospitalization or death.

The superiority of RVFW-LS/PASP and RVEF/PASP over other echocardiographic surrogates of RVPAC might be explained by the very superiority of RVFW-LS and 3D RVEF over other conventional parameters of RV function. Carluccio et al. showed in their previous study that RVFW-LS is a better outcome predictor in left-sided HF than TAPSE [4] and GLS-RV [31]. We reported in a recent article [5] that 3D RVEF is an independent predictor of adverse events in DCM, outperforming other RV functional indices in this clinical setting. Compared to other parameters assessing RV longitudinal function (such as TAPSE and S wave velocity), RVFWLS detects subtle myocardial abnormalities and is relatively angle-independent [32]. Moreover, compared to GLS-RV, it is considered more specific for the RV [33] because GLS$\mathrm{RV}$ also integrates the motion of the IVS (common to both ventricles), and LV dysfunction might restrict its value [31]. 3D RVEF is particularly useful for RV assessment because it is the only parameter that overcomes $2 \mathrm{D}$ geometric assumptions and integrates the longitudinal, radial, and anteroposterior components of RV contraction [33]. The fact that LVEF was not a predictor of rehospitalizations in our study might be partly explained by the fact that it was severely reduced throughout the entire cohort, with its values within a narrow range. Previous data suggested that the ability of LVEF to predict rehospitalizations is not related to its absolute value, but rather to its trajectory over time and its correlation with the patient's hemodynamic and neurohormonal status [34].

\section{Implications}

This echocardiographic study is the first to perform a pointby-point comparison of multiple methods to estimate RVPAC and of their prognostic role in DCM. Our results highlight the importance of evaluating the RV and pulmonary circulation as an integrative unit improving prognostic prediction accuracy when combining parameters of RV function with PASP. Among different non-invasive surrogates for RVPAC, RVFW-LS/PASP and RVEF/PASP — both derived from innovative echocardiographic techniques - are independent predictors for HF rehospitalizations in DCM and might improve risk stratification in clinical practice.

\section{Limitations}

The main limitation of this study is the lack of concomitant validation of echocardiographic RVPAC with gold-standard invasive measurements. However, the surrogates we chose in this study have been previously utilized and validated with catheterization-derived RVPAC $[10,24]$. Another limitation comes from the small sample size and the single-center design. Last but not least, a good acoustic window and a regular heart rhythm are mandatory to accurately interpret 3D acquisitions and, by excluding patients with atrial fibrillation or poor window, our data became vulnerable to selection bias. This might be particularly important for atrial fibrillation patients because a recent study found that the prevalence of this arrhythmia among patients with DCM is as high as 30\% [35]. Further research will be needed to establish if these results can be extrapolated to larger cohorts.

\section{CONCLUSION}

We found that RVFW-LS/PASP and RVEF/PASP are independent predictors for HF rehospitalization in patients with DCM. This study reinforces the idea that the RV and pulmonary circulation are intertwined components of a functional unit, that right ventriculo-vascular interaction has an independent prognostic role in left-sided HF and that RVPAC assessment, which can also be done non-invasively, might improve risk stratification, should it be validated in further research. 


\section{Article information}

Acknowledgments: This work was supported by CREDO Project - ID: 49182, financed by the National Authority of Scientific Research and Innovation, on behalf of the Romanian Ministry of European Funds, through the Sector Operational Program "Increasing of Economic Competitiveness", Priority Axis 2, Operation 2.2.1 (SOP IEC -A2-0.2.2. 1-2013-1) co-financed by the European Regional Development Fund. Conflict of interest: None declared.

Open access: This article is available in open access under Creative Common Attribution-Non-Commercial-No Derivatives 4.0 International (CC BY-NC-ND 4.0) license, allowing to download articles and share them with others as long as they credit the authors and the publisher, but without permission to change them in any way or use them commercially. For commercial use, please contact the journal office at kardiologiapolska@ptkardio.pl.

\section{REFERENCES}

1. Nagata Y, Wu VCC, Kado Y, et al. Prognostic value of right ventricular ejection fraction assessed by transthoracic 3D echocardiography. Circ Cardiovasc Imaging. 2017; 10(2), doi: 10.1161/CIRCIMAGING.116.005384, indexed in Pubmed: 28174197.

2. Seo J, Jung $\mathrm{IH}$, Park JH, et al. The prognostic value of $2 \mathrm{D}$ strain in assessment of the right ventricle in patients with dilated cardiomyopathy. Eur Heart J Cardiovasc Imaging. 2019; 20(9): 1043-1050, doi: 10.1093/ehjci/jez015, indexed in Pubmed: 30796431.

3. Fine NM, Chen L, Bastiansen PM, et al. Outcome prediction by quantitative right ventricular function assessment in 575 subjects evaluated for pulmonary hypertension. Circ Cardiovasc Imaging. 2013; 6(5): 711-721, doi: 10.1161/CIRCIMAGING.113.000640, indexed in Pubmed: 23811750.

4. Carluccio E, Biagioli $P$, Alunni $G$, et al. Prognostic value of right ventricular dysfunction in heart failure with reduced ejection fraction: superiority of longitudinal strain over tricuspid annular plane systolic excursion. Circ Cardiovasc Imaging. 2018; 11(1): e006894, doi: 10.1161/CIRCIMAGING.117.006894, indexed in Pubmed: 29321212.

5. Vijîiiac A, Onciul S, Guzu C, et al. The prognostic value of right ventricular longitudinal strain and 3D ejection fraction in patients with dilated cardiomyopathy. Int J Cardiovasc Imaging. 2021; 37(11): 3233-3244, doi: 10.1007/s10554-021-02322-z, indexed in Pubmed: 34165699.

6. Guazzi M, Dixon D, Labate V, et al. RV contractile function and its coupling to pulmonary circulation in heart failure with preserved ejection fraction: stratification of clinical phenotypes and outcomes. JACC Cardiovasc Imaging. 2017; 10(10 Pt B): 1211-1221, doi: 10.1016/j.jcmg.2016.12.024, indexed in Pubmed: 28412423.

7. Ikonomidis I, Aboyans V, Blacher J, et al. The role of ventricular-arterial coupling in cardiac disease and heart failure: assessment, clinical implications and therapeutic interventions. A consensus document of the European Society of Cardiology Working Group on Aorta \& Peripheral Vascular Diseases, European Association of Cardiovascular Imaging, and Heart Failure Association. Eur J Heart Fail. 2019; 21(4): 402-424, doi: 10.1002/ejhf.1436, indexed in Pubmed: 30859669.

8. Monge García MI, Santos A. Understanding ventriculo-arterial coupling Ann Transl Med. 2020; 8(12): 795, doi: 10.21037/atm.2020.04.10, indexed in Pubmed: 32647720.

9. Brimioulle $S$, Wauthy $P$, Ewalenko $P$, et al. Single-beat estimation of right ventricular end-systolic pressure-volume relationship. Am J Physiol Heart Circ Physiol. 2003; 284(5): H1625-H1630, doi: 10.1152/ajpheart.01023.2002, indexed in Pubmed: 12531727.

10. Schmeisser A, Rauwolf T, Groscheck T, et al. Pressure-volume loop validation of TAPSE/PASP for right ventricular arterial coupling in heart failure with pulmonary hypertension. Eur Heart J Cardiovasc Imaging. 2021; 22(2): 168-176, doi: 10.1093/ehjci/jeaa285, indexed in Pubmed: 33167032.

11. Nochioka K, Querejeta Roca G, Claggett B, et al. Right ventricular function, right ventricular-pulmonary artery coupling, and heart failure risk in 4 US communities: the Atherosclerosis Risk in Communities (ARIC) study. JAMA Cardiol. 2018;3(10): 939-948, doi: 10.1001/jamacardio.2018.2454, indexed in Pubmed: 30140848.

12. lacoviello M, Monitillo F, Citarelli G, et al. Right ventriculo-arterial coupling assessed by two-dimensional strain: A new parameter of right ventricular function independently associated with prognosis in chronic heart failure patients. Int J Cardiol. 2017; 241: 318-321, doi: 10.1016/j. ijcard.2017.04.051, indexed in Pubmed: 28479093.

13. Pestelli G, Fiorencis A, Trevisan F, et al. New measures of right ventricle-pulmonary artery coupling in heart failure: An all-cause mortality echocardiographic study. Int J Cardiol. 2021; 329: 234-241, doi: 10.1016/j. ijcard.2020.12.057, indexed in Pubmed: 33359279.

14. Pinto YM, Elliott PM, Arbustini E, et al. Proposal for a revised definition of dilated cardiomyopathy, hypokinetic non-dilated cardiomyopathy, and its implications for clinical practice: a position statement of the ESC working group on myocardial and pericardial diseases. Eur Heart J. 2016; 37(23): 1850-1858, doi: 10.1093/eurheartj/ehv727, indexed in Pubmed: 26792875.

15. Lang RM, Badano PL, Mor-Avi V, et al. Recommendations for Cardiac Chamber Quantification by Echocardiography in Adults: An Update from the American Society of Echocardiography and the European Association of, Cardiovascular Imaging. Eur Heart J Cardiovasc Imaging. 2016; 17(4): 412, doi: 10.1093/ehjci/jew041, indexed in Pubmed: 26983884.

16. Mitchell C, Rahko PS, Blauwet LA, et al. Guidelines for Performing a Comprehensive Transthoracic Echocardiographic Examination in Adults: Recommendations from the American Society of Echocardiography. J Am Soc Echocardiogr. 2019;32(1): 1-64, doi: 10.1016/j.echo.2018.06.004, indexed in Pubmed: 30282592.

17. Rudski LG, Lai WW, Afilalo J, et al. Guidelines for the Echocardiographic Assessment of the Right Heart in Adults: A Report from the American Society of Echocardiography Endorsed by the European Association of Echocardiography, a Registered Branch of the European Society of Cardiology, and the Canadian Society of Echocardiography. J Am Soc Echocardiogr. 2010; 23(7): 685-713, doi: 10.1016/j.echo.2010.05.010, indexed in Pubmed: 20620859.

18. Muraru D, Onciul S, Peluso D, et al. Sex- and method-specific reference values for right ventricular strain by 2-dimensional speckle-tracking echocardiography. Circ Cardiovasc Imaging. 2016; 9(2): e003866, doi: 10.1161/CIRCIMAGING.115.003866, indexed in Pubmed: 26860970.

19. Badano LP, Kolias TJ, Muraru D, et al. Standardization of left atrial, right ventricular, and right atrial deformation imaging using two-dimensional speckle tracking echocardiography: a consensus document of the EAC$\mathrm{VI} / \mathrm{ASE} /$ Industry Task Force to standardize deformation imaging. Eur Heart J Cardiovasc Imaging. 2018; 19(6): 591-600, doi: 10.1093/ehjci/jey042, indexed in Pubmed: 29596561.

20. Muraru D, Spadotto V, Cecchetto A, et al. New speckle-tracking algorithm for right ventricular volume analysis from three-dimensional echocardiographic data sets: validation with cardiac magnetic resonance and comparison with the previous analysis tool. Eur Heart J Cardiovasc Imaging. 2016; 17(11): 1279-1289, doi: 10.1093/ehjci/jev309, indexed in Pubmed: 26647080.

21. Tello K, Wan J, Dalmer A, et al. Validation of the tricuspid annular plane systolic excursion/systolic pulmonary artery pressure ratio for the assessment of right ventricular-arterial coupling in severe pulmonary hypertension. Circ Cardiovasc Imaging. 2019; 12(9), doi: 10.1161/circimaging.119.009047.

22. Bosch L, Lam CSP, Gong L, et al. Right ventricular dysfunction in left-sided heart failure with preserved versus reduced ejection fraction. Eur J Heart Fail. 2017; 19(12): 1664-1671, doi: 10.1002/ejhf.873, indexed in Pubmed: 28597497.

23. Deaconu S, Deaconu A, Scarlatescu A, et al. Ratio between right ventricular longitudinal strain and pulmonary arterial systolic pressure: novel prognostic parameter in patients undergoing cardiac resynchronization therapy. J Clin Med. 2021; 10(11), doi: 10.3390/jcm10112442, indexed in Pubmed: 34072825.

24. Aubert R, Venner C, Huttin O, et al. Three-Dimensional echocardiography for the assessment of right ventriculo-arterial coupling. J Am Soc Echocardiogr. 2018; 31(8): 905-915, doi: 10.1016/j.echo.2018.04.013, indexed in Pubmed: 29958760.

25. Jone PN, Schäfer M, Pan Z, et al. Right ventricular-arterial coupling ratio derived from 3-dimensional echocardiography predicts outcomes in pediatric pulmonary hypertension. Circ Cardiovasc Imaging. 2019; 12(1): e008176, doi: 10.1161/CIRCIMAGING.118.008176, indexed in Pubmed: 30632388.

26. Vonk Noordegraaf A, Westerhof BE, Westerhof N. The relationship between the right ventricle and its load in pulmonary hypertension. J Am 
Coll Cardiol. 2017;69(2):236-243, doi: 10.1016/j.jacc.2016.10.047, indexed in Pubmed: 28081831.

27. Todaro MC, Carerj S, Zito C, et al. Echocardiographic evaluation of right ventricular-arterial coupling in pulmonary hypertension. Am J Cardiovasc Dis. 2020; 10(4): 272-283, indexed in Pubmed: 33224574

28. Zaidi A, Knight DS, Augustine DX, et al. Echocardiographic assessment of the right heart in adults: a practical guideline from the British Society of Echocardiography. Echo Res Pract. 2020; 7(1): G19-G41, indexed in Pubmed: 32105053.

29. Adir Y, Guazzi M, Offer A, et al. all investigators. Different correlates but similar prognostic implications for right ventricular dysfunction in heart failure patients with reduced or preserved ejection fraction. Eur J Heart Fail. 2017; 19(7): 873-879, doi: 10.1002/ejhf.664, indexed in Pubmed: 27860029.

30. Brand $A$, Bathe $M$, Oertelt-Prigione $S$, et al. Right heart function in impaired left ventricular diastolic function: $2 \mathrm{D}$ speckle tracking echocardiography-based and Doppler tissue imaging-based analysis of right atrial and ventricular function. Echocardiography. 2018;35(1):47-55, doi: 10.1111/echo.13745, indexed in Pubmed: 29106754.
31. Carluccio E, Biagioli P, Lauciello R, et al. Superior prognostic value of right ventricular free wall compared to global longitudinal strain in patients with heart failure. J Am Soc Echocardiogr. 2019; 32(7): 836-844.e1, doi: 10.1016/j.echo.2019.02.011, indexed in Pubmed: 30979539.

32. Lopez-Candales A, Hernandez-Suarez DF. Strain imaging echocardiography: what imaging cardiologists should know. Curr Cardiol Rev. 2017; 13(2): 118-129, doi: 10.2174/1573403X12666161028122649, indexed in Pubmed: 27799029.

33. Sciaccaluga C, D'Ascenzi F, Mandoli GE, et al. Traditional and novel imaging of right ventricular function in patients with heart failure and reduced ejection fraction. Curr Heart Fail Rep. 2020; 17(2): 28-33, doi: 10.1007/s11897-020-00455-1, indexed in Pubmed: 32130642.

34. Kalogeropoulos AP, Butler J. Left ventricular ejection fraction in patients with acute heart failure: a limited tool? Rev Esp Cardiol (Engl Ed). 2017; 70(5): 318-319, doi: 10.1016/j.rec.2016.11.029, indexed in Pubmed: 28073644.

35. Dziewięcka E, Gliniak M, Winiarczyk M, et al. The burden of atrial fibrillation and its prognostic value in patients with dilated cardiomyopathy. Kardiol Pol. 2020; 78(1): 37-44, doi: 10.33963/KP.15046, indexed in Pubmed: 31686668. 